\title{
NAS: A DISTINCT WAY TO STORE AFFORDABLY
}

\author{
Joy Singh \\ Student at Manav Rachna International \\ Institute of Research and Studies, Faridabad, \\ 121003, Haryana, India
}

\begin{abstract}
The requirement for large storage repositories has increased multi times in recent times; new type of network storage has become important for large data storage. The network storage system must have high capacity and availability. Recent trends in technology have alleviate the need to eliminate direct attached storage to aid more flexible storage system. Information Storage Systems are the most essential members like humans on which a modern company rests. Network based storage combined with smart storage management like DAS (Directly Attached Storage), NAS (Network Attached server), and SAN (Storage Attached Network). This paper focuses on NAS, its applications, comparison and construction.
\end{abstract}

\section{INTRODUCTION}

Due to the development of various fields like Iot, network economy, personalised ads etc. Huge amount of data is generated within seconds and require an storage entity with high capacity, availability and scalability. SAN and NAS storage systems introduced a new and efficient way to process as well as store data efficiently. Use of open standards in makes them a constant evolving with each user making a contribution of their own. Information storage systems run the modern world countries and help in generating new economy known as network economy. Data needs to be available to every needy, at any time and at any place. As a result, the eccentricity is beginning to shift from a high processing power to more storage. Processing is merely a form changer and storage system can provide continuous accessibility to information, with protection against unauthorised access, piracy and many a times redundancy is also taken care of. Scalability and performance is not compromised while delivering all above functions. Data storage has become one of the main problems in the development of newer network because new network will put an extra load on the existing data storing structures and all data need some physical place to store it is becoming more of challenge to store high amount of data at small places. With the high capacity, high I/O transfer speed, and high system availability, a network storage device can be used for information access as well as data sharing
Sudhanshu Kumar Jha

Student at Manav Rachna International

Institute of Research and Studies, Faridabad, 121003, Haryana, India

by multiple users. One survey showed that in the last one decade (2010-2020), the data universe have grown by a factor of 400, from 130 Exabyte's to 45,000 Exabyte's, or 45 trillion gigabytes with more than 3.3 million data flowing across internet each passing second.

Computing is operating upon information. Information stored in storage media is used by applications running on a server or by a user for reference. Often it is an asset for which accompany survives. It cannot be considered wrong to say that each nation's security also depends on data storages architectures as it may hold records of officer's secret files and other stuff related to military equipment. It is the currency of business in modern world and now will continue to do so even in future. To ensure that any business gives the best, they must have access to accurate information with security. The management and protection of information is very vital. The investment in IT hardware, software, service, telecommunication and staff are considered as the - foundation and infrastructure of the digital universe and communications. Investment in storage management, security, big data, and cloud computing will grow very fast. Any breach in this infrastructure can bring about a devastation to companies as well as to a nation.

Till the emergence of new storage architecture, the storage area network technique provides a solution to the problem of achieving information integration with sharing; while providing manageability, accessibility and high security. The SAN uses a network type of storage structure as well as difference between data storage and computation. Features like dynamic addressing, long-distance transmission, significant I/O (input/output) speed, and data sharing. In layman's terms, Network Storage is all about storing data using a method by which it can be made securely available to clients on the network at any point of use. A NAS system is a special-purposed device designed to provide clients with files on a local network, with advantages like sharing files in a hetero-architecture, achieved using the current LAN architecture, operation, and network adaptation at low cost. All these features make it better storage system. 


\section{DIRECT ATTACHED STORAGE} (DAS)

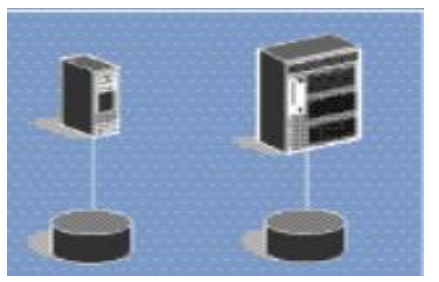

Storage types like disk are directly attached by a cable to the computer processor. (HDD (Hard Disk Drive), SSD (Solid State Drive) inside a PC or a single server are one of the types of DAS). I/O requests (commands) or simple query injections access devices directly.

\section{STORAGE ATTACHED NETWORK}

Dedicated network is the requirement. Like DAS, I/O requests access devices directly. Mostly Fibre optics is used for providing high speed processors and storage communications on the network.

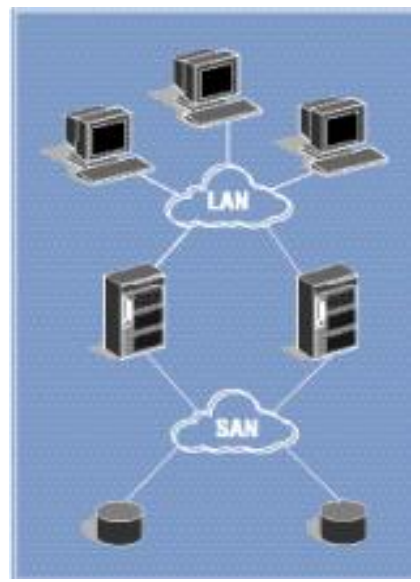

The major potential benefits of a SAN can be categorized as:

- Access: Storage processor distance is covered generally by optical media providing high availability (because I/O traffic is transferred from LAN to a dedicated network,). Also, a number of processors can be connected to the same storage device compared to built-in device attachment facilities.

Consolidation: When very few devices on a network compensate for many devices they are known as pooling SANs provide high level scalability through software but hardware acting as single unit is also possible by the same architecture. A single point of control is there to allow users to use multiple SANs. Control over which hosts can see which storage using zoning and LUN masking is being implemented in real time. -Protection: SAN comes with its own backup facilities without the use of LAN so it can be faster than LAN at times while server-free backups can let disk storage writing directly to tape without processor overhead. - Data Sharing: Sharing data is one of the main reasons that these storage systems are designed. It uses a tailored network usually based on Fibre optics channel; the initial cost to implement a SAN is higher than any other storage systems as it require special hardware and software for management and provide potential benefits. Additionally, organizations require skills to manage this sophisticated yet simple technology. However, an analysis may justify the cost due to the long-term lower Total Cost compared to any alternate approach.

\section{NETWORK ATTCHED STORAGE}

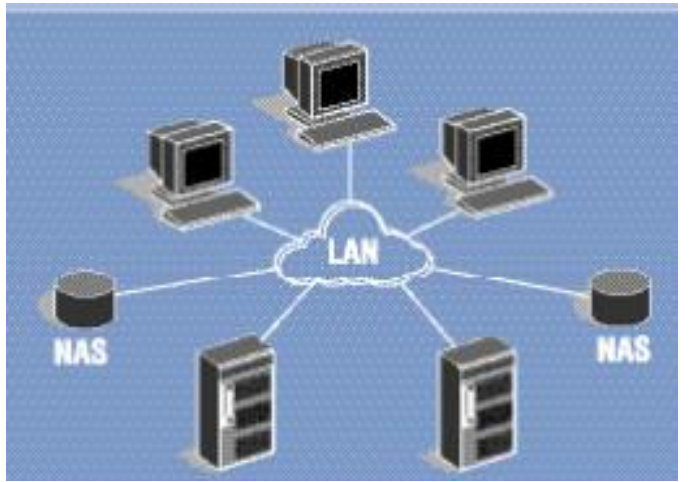

NAS is an algorithm or set of protocols that store data on various interconnected network. Each device has individual IP address and can easily be accessed by clients via a server acting like gateway to the data or in some cases allows the device to be accessed by users without a mediator in between. Benefit of NAS lies in its environment with many servers running different operating systems, centralised storage of data with security, management, backup of the data, accessing File-level data. It represents file level interface to outside and block level interface for storage subsystems.

\section{NAS vs. DAS}

- DAS is designed for use with single, isolated processor with low initial cost. While NAS attaches various storage elements for file sharing using low cost Ethernet connected networks. Installation is quicker in DAS while little amount of necessary network information is required for NAS installation.

- Storage capacity is automatically assigned to users with appropriate demand in NAS while full capacity is available to user with DAS.

- DAS has single storage device connected to a single processor. 


\section{International Journal of Engineering Applied Sciences and Technology, 2020 Vol. 5, Issue 6, ISSN No. 2455-2143, Pages 341-348 \\ Published Online October 2020 in IJEAST (http://www.ijeast.com)}

NAS one or more processors connected to one or more storage devices.

- Security in DAS regards physical safety. If data is stolen physically no retrieval is possible until drive is found. In a NAS the security is designed to keep away all unauthentic users via. a password or in case of data theft cloud based services for data recovery or backup can be used at all times.

- Medium of connection in DAS is physical wiring connecting storage and processor. While in NAS media is accessed by an Ethernet protocol unique to that media regardless of the attached devices. A protocol is defined as a set of rules for information exchange between two objects. For a computer, this specifies the format and sequence of electronic messages necessary for communication from both sides. In storage-processor connections, the following media and associated protocols are used in common. All standards followed are industry ranked as well as open.

Common technology in use-Ethernet: Ethernet arose to create LAN in late $20^{\text {th }}$ century. General bandwidths are in range of (10Mbps- 100Mbps) sometimes reaching $1 \mathrm{Gbps} .3$ Ethernet is a transportation media and TCP/IP is its administrative rule. - Fibre Channel: Optic fiber Channel was developed in late 1990s and has become most used both in storage (for both SAN and DAS) as well as communication (both short and long distance). Bandwidth is generally $100 \mathrm{MBps}$ to $200 \mathrm{MBps}$ reaching speeds in GBps oftenly.

- Parallel SCSI (Small Computer Systems Interface): (Pronounced "scuzzy"). It has been a constantly evolving technology since its development in the 1980s. Bandwidths are 40MBps (common name- UltraSCSI), 80MBps (common name- Ultra2 SCSI), and 160MBps (Ultra160 SCSI).

Max distance covered $=25 \mathrm{~m}$

So it is efficient for direct attach storage, especially when storage and processors lie in very close proximity of a cabinet, but not well-suited for networking. -SSA (Serial Storage Architecture): It is a media technology for high-performance and connecting disks together inside some disk systems. Bandwidth is 160MBps (MBps=megabytes/second,

Mbps=megabits/second).
SQL processing uses specific protocols that run at the topmost level above the media protocols. (In the case of Ethernet, protocols generally run at an IP protocol stack.)

Following are protocols supported on midrange platforms.

- $\underline{\mathrm{SCSI}}$ (Small Computer Systems Interface): This protocol is most used in midrange. A SCSI SQL command can return data from a specific location on a disk drive, or a tape library toggling between specific cartridges. SCSI is a "block level" protocol, or block-I/O, because SCSI commands specify particular blocks (sectors) on a specific disk. Initially, SCSI commands were sent through "parallel SCSI" only. Now SCSI commands are executed over different types of transfer mediums like Optic Fibre Channel, SSA, Ethernet and parallel SCSI.

-NFS (Network File System): It is a file-level protocol to access and share data. It is deviceindependent as it might be just reading the initials of characters from a file, without the location of the data on the device. It's origins can be traced in UNIX world.

- CIFS (Common Internet File System, often pronounced "siffs"): Again a basic file standard protocol to access as well as share data. This protocol is device-independent as in NFS; a CIFS command can only request reading the initial Eighty (80) characters from a file, without knowing the location of data storage on the disk. CIFS traces its origins in Microsoft Windows NT world. Due to SCSI (a type of block-I/O queries), storage volumes on disks become visible to the servers. With NFS and CIFS (file-reading/writing), files attached to processors become visible, but disk volume occupied by those files is unknown to those processors.

In DAS storage is attached by a cable to the processor directly. The media can be any (like Optic Fibre Channel, SCSI, SSA, and Ethernet).

In NAS TCP/IP based network (LAN or WAN) device is operated using CIFS and NFS - special protocols are there for file access and sharing. A NAS device is also known with names like file server, filer or NAS appliance. It addresses a NFS or CIFS request on a network and an internal processor forwards that request to the SCSI block-commands to access the appropriate device only visible to the NAS device.

\section{NAS vs. SAN}

SAN, storage device designed for performance and scalability. Major potential benefits include support for high-speed Optical Fibre Channel media which is used for data traffic, multiple disk management like a set of multipurpose storage device with a single point of control with backup facilities that 
reduce server and LAN utilization and with wide industry support.

NAS

has easy management and file sharing by using low cost Ethernet networks. Installation is fast and simple.

In SAN a dedicated network is used for storage devices thus providing multi connection for processors and storage devices on the network. The most common media is Optical Fibre Channel, but Ethernet is also emerging as a useful media.

A NAS device attached to a TCP/IPbased network (LAN or WAN), and accessed using CIFS and NFS (Near Field Communication)—-specialized I/O protocols for file access and sharing. A NAS device is sometimes also called as a file server, or "filer" or "NAS appliance". It receives a request through the network. The internal processor then requests the SCSI block to accesses the appropriate device only visible to the NAS product itself.

\section{Basic comparison}

1. Easy installation: NAS is easy to install and manage when compared to SAN. NAS appliances need no additional network as they can be installed on an existing LAN/WAN network. NAS manufacturers quote that it can be installed and be ready for use in 30 minutes. (Customization takes additional time). NAS storage can be accessed quickly, without volume definitions or special device drivers on disks. While a SAN takes planning, designing of an Optical Fibre Channel network and selection/installation of SAN management software into account. 2. Backup: Most NAS appliances in current world include a snapshot kind of backup facility, to backup data on a HDD or SSHD only reducing the application downtime. While in SAN only select disks or storage management packages use this facility.

Resource pooling: NAS allows pooling of appliances. The NAS device can be configured with systems, with multiple set of disk volumes. File handling of various users is managed by the file system space within is allotted as required. This is more efficient than buying separate disk for each user, which often leads to unequal space distribution among users with some having large spaces while others low. So pooling a NAS minimizes the manual assignment of the disk capacity among users during real time use. However, NAS pooling is already within a compatible NAS appliance, and there is very little sharing of resources between appliances if needed. This increases costs and management of the whole system as the number of NAS nodes grow significantly. But all devices on a SAN can be collaborated easily. As total capacity grows, a SAN remains easy to manage while becoming more cost effective.
File sharing: NAS provides file sharing, but with technology like SANergy, a SAN becomes capable of doing this as well. Many a times a NAS is installed not for file sharing, but due to its ease of installation and management. 5. Performance: How do NAS and SAN perform? It may depend on the particular configuration, but SAN is faster.

This is mainly due to: - SAN use a dedicated network (similar to a NAS). SAN network speed (100MBps Fibre Channel is more common).

- Host overhead0- Optical Fibre Channel protocol handling is done in host bus adapter; while TCP/IP protocol runs on host software and adds up considerable overhead. There is research being done to reduce TCP/IP protocol handling to host bus adapters, which will help in reducing processor overhead problem. For lower amount of transfers, NAS and SAN both perform equally well. Today, however, for very large data NAS will generally not scale as well as SAN in performance. It is not known where the "even" point or the point of equal performance in terms of request handling is, but NAS devices can handle several thousand queries per second with response time (e.g., under 10 milliseconds average for small random I/Os). To summarize the comparison between NAS and SAN, NAS is generally less scalable as well as grandiose than a SAN,

\section{NAS GATEWAYS}

A NAS gateway is enhanced form of NAS where it provides the features of a NAS without integrating the disk storage or appliance. The disk storage is externally attached to the gateway, and can also work with SAN or direct attach as a standalone offering. Gateway accepts a file read and write request (using NFS or CIFS protocols) and transmits that to a Small Computer Systems Interface (SCSI) block for accessing the external attached disk storage. Using gateway as an approach for file sharing offers the benefits of a conventional NAS appliance, with additional advantages like-Increase in choice of disk types. -Increase in capability (large read, cache writing or remote copy functions) -Increase in disk scalability (in comparison to the capacity limits of an integrated NAS appliance) - Ability to preserve along with value enhancement of select installed disk systems by file sharing - Ability to share file with block-I/O on the same disk system. Disk capacity of SAN can be shared between gateway and non-gateway. So a gateway can be viewed as a NAS or SAN hybrid, increasing flexibility while lowering costs (capacity often go unutilized if it were permanently dedicated to a NAS or SAN appliance). 


\section{PROTOCOLS OF NAS}

\section{NFS protocol}

NFS is a distributive type of file system protocol that allows the user to access files via the client network computer which is similar to accessing the local storage. NFS similarity to other protocol creates an Open Network Computing Remote Procedure Call (ONCRPC) system. 2.0 of the protocol originally operated on UDP. NFS version 2 of the protocol kept the server side stateless along with locking while 3.0 supports TCP as transport protocol in addition to support for 64-bit file sizes and offsets, asynchronous writes on the server to improve writing performance and supports additional file attributes.

Parallel NFS or pNFS is the standard protocol for parallel Input or output access of storage. This shows that pNFS can achieve the primary goal of aggregating the Input or Output bandwidth from multi storage servers. Matthias Grawinkel et al. presented the extension integrating the client's ability to provide Input or Output devise to metadata server.

\section{B. CIFS protocol:}

Common Internet File System (CIFS) is the protocol used by computer users to share files through intranet for local and the Internet for worldwide. CIFS is a modern speech of server electronic message block (SMB). It operates in application layer of OSI protocol. It is used for providing common file access along with peripherals such as printers, serial ports, monitors and other communication between nodes on a network. CIFS provides communication mechanism with authentication facility.

CIFS shares the information with the interconnected computers by a series of messages which can be broadly divided as, Connection Establishment Messages, Namespace and File Manipulation messages.

CIFS supported platforms -Windows OS, UNIX, Mac, IBM LAN, MS-Net, etc. CIFS satisfy Hypertext Transfer Protocol Secured (HTTPS) in modern day file shares. Older protocols, like FTP, CIFS only ensured data integrity by preventing conflicts. When users share files using only file lock method it only one file copy gets active at a time to avoid data corruption. CIFS protocols have been designed to work at slow speed connections too. CIFS provides security for files by using secure transmission of files and authorisation before one can access the files. CIFS supports Unicode file names are used so that one has a large character set to choose from and keep the desired name. CIFS performs better because of its integration with Operating System and thus promotes maximum available system performance. CIFS has Global File Names support so the users need not mount the remote file system, remote files can be accessed directly with global names.

\section{CASE STUDIES}

The table below identifies a few simple scenarios on what may be effective storage connectivity approaches.

\begin{tabular}{|l|l|}
\hline SITUATION & POSSIBLE SOLUTION \\
\hline $\begin{array}{l}\text { PLACE-Organisation } \\
\text { PROBLEM- Low no. of servers so considering of } \\
\text { direct attach }\end{array}$ & $\begin{array}{l}\text { NAS is the most optimum solution. } \\
\text { The reason to support NAS is its ability to communicate } \\
\text { wirelessly as well as direct attach can favour the } \\
\text { organisation in long term goal by features like snap } \\
\text { backup in the cloud as well physical safety to the drives } \\
\text { that keep valuable information. }\end{array}$ \\
\hline $\begin{array}{l}\text { PLACE- Organisation } \\
\text { PROBLEM- Empty LAN bandwidth }\end{array}$ & $\begin{array}{l}\text { NAS or SCSI } \\
\text { These are the most commonly used when intra } \\
\text { connected or short connections are preferred. SCSI is } \\
\text { best when data sharing is not an aspect of the problem. }\end{array}$ \\
\hline
\end{tabular}


PLACE- Organisation

PROBLEM- Existing servers of IBM and want to do a bit of data sharing
SANergy or a NAS gateway

These are the best because of preservation nature of disk as opposed to buying or upgrading to new disk.

\section{CREATION OF LOCAL NAS USING RASBERRY PI 4 -B}

\section{Requirements}

- 2 USB drives (External, minimum requirement), e.g. WD HDD or SSD

- USB 3.0 compatible hub

- Gigabit Ethernet (best for this use)

- Constant power supply(inverter with battery)

\section{Step 01: Storage Requirement Analysis}

The most important decision that should be taken beforehand is the amount odf extra space as required by us and the buying the disks accordingly though it is upgradable at any instance of time with little modifications to the code written. Classical rotating drives give us low cost with high capacity than a SSD or SSHD. Raspberry Pi 4-B has built in USB 3.0, to make sure your external USB drives take advantage of the extra speed provided. For additional layer of protection, one need to multiply (here 2) the number of drives to assure data safety. We are using two 1 terabyte of storage. For reliable power one can add a USB 3.0 hub.

Step 02: Preparing the Operating System The Raspbian Buster Lite should be downloaded and burnt to a microSD card.

Steps after booting

Enable the $\underline{\mathrm{SSH}}$ by running sudo raspi-config (ii) Then select Interfacing Options > SSH. If you want, configure the Wi-Fi now, but for a decent NAS one should be using the high-speed Gigabit Ethernet port.

(iii) End step is to change one's password and beneath Network Options, the Hostname (the NAS's network name) should be changed which is solely on user's discretion. Ours has been changed to 'nas', so that the network address becomes 'nas.local'. Finally, the latest update should be checked with the following commandsudo apt update \&\& sudo apt -y upgrade, then reboot.

\section{Step 03: Adding Storage}

USB 3.0 powered hub should be connected to the Raspberry Pi 4-B.

Connect the disk drives.

Then the system is given sometime to check the disk's status, following is entered afterwards:

lsblk
This command reveals about the devices connected to the system. The device named with mmcblk0 is the microSD card holding our previously downloaded Raspbian OS. If someone has two USB disks installed and running, he should see 'sda' and 'sdb' (Storage Device A and Storage Device B). Further storage devices are named alphabetically.

\section{Step 04: Preparing the drives}

Next we do the partition of the drives for the Raspbian to understand where to store data on them. For this we use fdisk.

\section{sudo fdisk /dev/sda}

When asked for a command, ' $n$ ' should be entered for new partition. (If an error is detected during this event telling that a partition already exists, press ' $d$ ' to delete it - this will eliminate all data on the disk!) For creating primary partition enter ' $p$ '. A series of questions will asked about various sectors. One should not panic and keep calm as most of them are irrelevant to daily or personal use. Only keep pressing ENTER until 'Created a new partition' appears as described earlier most of the options asked are default ones. Next we type 'w' (to write the changes to the disk if any).

fdisk will exit now. The process must be reppeated for the second drive too by entering the following:

\section{sudo fdisk $/ \mathrm{dev} / \mathrm{sdb}$}

repeating the steps as above...

\section{Step 05: Creating RAID}

RAID or Redundant Array of Inexpensive Disks is a type of data protection method where multiplication over multiple disks is done to prevent data from damages. Multiple forms are available or in use today, but we're using the simplest one: RAID-1, also known as mirroring. If a disk fails, the NAS will still keep on running and you don't lose anything. Replace the failed if any disk as soon as possible and the array is 'rebuilt'.

To do this first of all, install RAID manager, mdadm; command for the following is:

\section{sudo apt install mdadm}

Now the mdadm is instructed to create the RAID-1 array:

sudo mdadm --create --verbose /dev/md0 level=mirror --raid-devices=2 /dev/sda1 /dev/sdb1 


\section{International Journal of Engineering Applied Sciences and Technology, 2020 Vol. 5, Issue 6, ISSN No. 2455-2143, Pages 341-348 \\ Published Online October 2020 in IJEAST (http://www.ijeast.com)}

Step 06: Mounting the drive

Raspbian can now see both physical disks as a single disk entity. Format and mount the newly formed virtual drive:

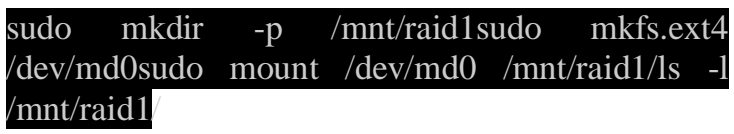

You should see one item: 'lost found'. This indicates RAID-1 system is operational. Next, do make sure that the drive is mounted whenever you boot.

\section{sudo nano /etc/fstab}

Add the line:

$/ \mathrm{dev} / \mathrm{md} 0 / \mathrm{mnt} / \mathrm{raid} 1 /$ ext4 defaults,noatime 01

Quit (CTRL+X, followed by Y), then following is run so the RAID array starts up correctly on boot:

sudo mdadm --detail --scan
letc/mdadm/mdadm.conf

After reboot you ought to have /mnt/raid1 ready to go.

\section{Step 07: The Samba! In play}

For sharing files on the existing network using the $\mathrm{SMB} / \mathrm{CIFS}$ protocol though the Raspbian version comes with a friendlier name of Samba, but it is not installed by default. Run the following:

\section{sudo apt install samba samba-common-bin}

If any further questions are asked, just do the default answer selection. Let us create a directory to allow all users its access:

\section{sudo mkdir /mnt/raid1/sharedsudo chmod -R 777 /mnt/raid1/shared}

Tell the Samba to share the directory on the network by editing the config file:

sudo nano /etc/samba/smb.conf

At the bottom, add the following:

[shared]path $=/ \mathrm{mnt} / \mathrm{raid} 1 /$ sharedwriteable $=$ Yescreat

e mask $=0777$ directory mask $=0777$ public $=$ no

Save (CTRL+X, followed by Y), then restart Samba:

\section{sudo systemctl restart smbd}

Step 08: Granting access

To give a user access to the files stored on the NAS, a special command needs to be set a Samba password. So, to grant access to the current user, 'pi':

\section{sudo smbpasswd -a pi}

A password (no need for it to be the same as our Raspberry Pi password) must be set for the purpose of authorization and data safety. After this, the guest or administrator user 'pi' can access the Samba share from Windows, macOS, or other Raspberry $\mathrm{Pi}$ devices, with the ability to read and write files.

To create additional guest users:

sudo adduser usernamesudo smbpasswd -a
username

...username is opted by user itself.

Step 9: Creation of home directories

If one wants to keep data private according to the preference of individual users it is possible by creating file directories private the respective user itself through RAID array:

mkdir /mnt/raid1/shared/usernamesudo chown -R
username /mnt/raid1/shared/usernamesudo chmod -
$\mathrm{R} 700 / \mathrm{mnt} /$ raid1/shared/username

Again, custom username is possible according to our wishes username. Now only that particular user can access that directory.

Step 10: Backup, backup, backup

RAID is not a backup system. It only provides a certain level reassurance against data redundancy, but is of no use in case of data loss or theft. If any drive fails, our system will demote to a 'degraded' state, where all data is at risk until replacement of disk appears. If any further devices become corrupt or go in paralyzed state then disaster will be our guest.

The ideal and most optimized solution is using a cloud storage service provider such as Google or Amazon to back everything up at somewhat far but secure place. Utilities like Rclone can sync entire directory structures onto many different providers' storage servers. A regular cron job can be created to make sure your data survives.

\section{Step 11: Don't interrupt!}

This project can be taken to a level beyond regular use and by creating a real server like feel by assigning its own UPS (Uninterrupted Power Supply). Sudden power cuts can spell disaster for Linux-based systems due to the way they handle files in memory. This may cause it to sleep forever. This battery backup safely keeps your Raspberry Pi and hub running in the event of a power cut. Many types of UPS can communicate their status to our Raspberry Pi over USB, so a safe shutdown can be triggered.

\section{CONCLUSION}

NAS can be expensive. For a cheaper way, thanks to store a lot of files to share with friends or family, 
Raspberry Pi 4 is right . It can't compete with Intelbased systems in terms of speed or features, but if you have some external USB disks lying around, it's a very affordable way to not only serve your data, but protect it also. So it has come to this- IS NAS for you? Well what is a NAS, anyway? A wellimplemented, network-attached memory device is usually a headless device (no keyboard or monitor) providing access to large amounts of knowledge from anywhere on your network. It must support and should be compatible for future availability and data retention. Which means that the server should work in all conditions especially any type of system failure and should be able to retain the data during any failure and the files stored on the server itself should be stored taking certain security measures using various suited protocols. In simpler terms, it's a box on which you dump all your movies, photos, music, and other stuff so you can get to it wherever and whenever without missing on protection.

\section{REFERENCES}

[1]. Amit Rakesh Shrivastava, Home server and nas using raspberry PI, 2017

\section{[2]. S.MOHANTY, NETWORK STORAGE AND} ITS FUTURE, 2010

[3]. Rhythm Kr Dasgupta, Wireless Media Server Using Raspberry Pi,2018

[4]. Gerard J. Holman: Design and Validation of Computer Protocols. Prentice Hall, 1991. t http://spinroot.com/spin/Doc/Book91.html

[5].https://in.pcmag.com/news/134535/how-toturn-a-raspberry-pi-into-a-nas-for-whole-home-filesharing, By Whitson Gordon, 2019

[6].https://www.ionos.com/digitalguide/server/conf iguration/raspberry-pi-nas/

[7]. Ijreeice.com

[8]. Freecomputerbooks.com

[9]. www-8.ibm.com
[10]. Bnrg.eecs.berkeley.edu

[11]. Ijcea.com

[12]. Ijcsit.com

[13]. Magpi.rasberrypi.org

[14]. www.diskdrive.com

[15]. Scribd.com

[16]. Maben.homeip.net 